\title{
Efecto agudo del ejercicio físico de intensidad moderada sobre las variables espirométricas en sujetos asmáticos broncodilatados. Prueba piloto
}

\author{
Juan Carlos Sánchez-Delgado ${ }^{1,2}$, Adriana Jácome-Hortua ${ }^{3}$, Clara Liliana Aguirre-Aguirre ${ }^{4}$, Adriana Angarita-Fonseca ${ }^{5}$
}

\section{RESUMEN}

Introducción: el broncoespasmo inducido por el ejercicio (BIE) ocurre entre el 70 y 80 \% de los asmáticos y su aparición es más frecuente al terminar una sesión de ejercicio físico, principalmente de intensidad vigorosa.

Objetivo: evaluar el efecto agudo que puede tener el ejercicio físico de intensidad moderada sobre las variables espirométricas en sujetos asmáticos broncodilatados.

Metodología: se realizó un estudio cuasi-experimental de tipo antes y después. Se incluyeron diez sujetos (edad promedio $=23 \pm 4$ años), los cuales fueron divididos en dos grupos: cinco sujetos asmáticos y cinco sanos. Los dos grupos realizaron 10 minutos de calentamiento, 20 minutos de ejercicio en banda sin fin al $60 \%$ de la frecuencia cardíaca de entrenamiento, seguido de 5 minutos de recuperación. La función pulmonar fue evaluada antes del ejercicio y 15 minutos después del ejercicio.

Resultados: se encontraron diferencias estadísticamente significativas entre grupos (con asma $6 \%$ vs. sin asma $-1 \%, p=0,03$ ) en los cambios pre y pos del ejercicio de la relación del volumen espiratorio forzado en el primer segundo (VEF1) sobre la capacidad vital forzada (CVF). EI análisis multivariado mostró que la CVF post ejercicio en el grupo con asma fue significativamente menor que el del grupo sin asma, ajustado por evaluación basal y masa corporal total.

1 Fisioterapeuta, MSc. PhD (c). Profesor programa de Fisioterapia, Facultad de Ciencias de la Salud, Universidad de Santander, Grupo de Investigación Fisioterapia Integral, Bucaramanga Colombia.

2 Profesor. Universidad Santo Tomás, Bucaramanga, Colombia.

3 Fisioterapeuta, MSc. Profesor programa de Fisioterapia, Facultad de Ciencias de la Salud, Universidad de Santander, Grupo de Investigación Fisioterapia Integral, Bucaramanga, Colombia.

4 Fisioterapeuta, Esp. MSc (c). Profesor programa de Fisioterapia, Facultad de Ciencias de la Salud, Universidad de Santander, Bucaramanga, Colombia.

5 Fisioterapeuta, MSc. PhD (c). Profesor programa de Fisioterapia, Universidad de Santander, Facultad de Ciencias de la Salud, Grupo de Investigación en Manejo Clínico - CliniUDES, Grupo de Investigación Fisioterapia Integral, Bucaramanga, Colombia.

Correspondencia: Juan Carlos Sánchez-Delgado; juancarlossanchezd@gmail.com

Recibido: abril 14 de 2018

Aceptado: septiembre 12 de 2018

Cómo citar: Sánchez-Delgado JC, Jácome-Hortua A, Aguirre-Aguirre CL, Angarita-Fonseca A. Efecto agudo del ejercicio físico de intensidad moderada sobre las variables espirométricas en sujetos asmáticos broncodilatados. Prueba piloto. latreia. 2019 Ene-Mar;32(1):7-15. D0l 10.17533/udea.iatreia.v32n1a01. 
Conclusión: el ejercicio de intensidad moderada en banda sin fin, no mostró diferencias clínicamente significativas sobre los cambios del pre y post ejercicio de las variables espirométricas estudiadas, puesto que estos cambios en VEF1 o CVF no superaron el $10 \%$ teniendo como referencia la evaluación basal.

\section{PALABRAS CLAVE}

Actividad Motora; Asma Inducida por Ejercicio; Composición Corporal; Espirometría; Obstrucción de las Vías Aéreas

\section{SUMMARY}

Acute effect of moderate-intensity exercise on spirometric variables in broncodilated asthmatic subjects. A pilot study

Background: Exercise-Induced Bronchospasm (BIE) occurs in $70 \%$ to $80 \%$ of asthmatics and its occurrence is more frequent at the end of a session of physical exercise, mainly of vigorous intensity.

Objective: To evaluate the acute effect of moderateintensity physical exercise on spirometric variables in asthmatic subjects.

Methodology: A before-and-after type quasi-experimental desiogn. Ten subjects were included (mean age $=23 \pm 4$ years), which were divided into two groups: five asthmatic subjects and five healthy subjects. Both groups performed 10 min of warm-up, 20 min of treadmill exercise at an intensity of $60 \%$ of the Heart Rate Reserve, and a final cool-down of 5 minutes. Lung function was assessed before and 15 minutes after exercise.

Results: There were statistically sionificant differences between groups (with asthma $=6 \%$ vs. without asth$\mathrm{ma}=-1 \%, \mathrm{p}=0.03$ ) in the pre-post-exercise changes of the ratio of the forced expiratory volume in one second and the forced vital capacity (FEV1/FVC). The multivariate analysis showed that post-exercise FVC in subjects with asthma was significantly lower than in subjects without asthma, after adjusting for the baseline assessment and total body mass.

Conclusion: Moderate-intensity exercise on a treadmill did not shows clinically significant differences on the changes pre-post exercise of the studied spirometric variables, since the changes on FEV1 or FVC did not exceed $10 \%$ having as reference the baseline evaluation.

\section{KEY WORDS}

Asthma, Exercise-Induced; Airway Obstruction; Body Composition; Motor Activity; Spirometry

\section{INTRODUCCIÓN}

EI asma es un trastorno bronquial que se presenta a cualquier edad y cuyas características principales incluyen una inflamación crónica acompañada de broncoespasmo de las vías aéreas. El porcentaje de la población afectada en adultos oscila entre el 3 y el $9 \%$ y en niños puede llegar al ser el doble. Esta condición puede ser exacerbada por factores ambientales, nutricionales y el ejercicio físico (EF), principalmente. Sus síntomas pueden ser variables e intermitentes, entre los que se comprenden sibilancias, disnea paroxística y tos, de mayor predominio nocturno (1-7).

La patogénesis del BIE implica alteraciones osmóticas y/o térmicas en las vías aéreas, causadas por cambios en el flujo de aire respirado durante el ejercicio y se describe como un estrechamiento agudo de las vías respiratorias, que puede ser de corta duración y reversible después de un periodo de ejercicio. Del 40 al $90 \%$ de los asmáticos padecen BIE, siendo superior esta prevalencia en quienes son atletas (8). Para su diagnóstico son necesarias pruebas de función pulmonar que evalúan la presencia $y$ gravedad de la obstrucción en las vías aéreas, así como el efecto del broncodilatador y del ejercicio sobre el calibre de las mismas. Las dos variables usadas para el diaognóstico son la relación entre la capacidad vital forzada (CVF) y el volumen espiratorio forzado en el primer segundo (VEF1), los cuales permiten establecer la presencia de una obstrucción bronquial; la segunda variable es el valor global de VEF1, que determina la gravedad de la obstrucción (9).

La mayoría de los estudios encontrados describen que las altas intensidades del ejercicio son uno de los principales factores desencadenantes del 
broncoespasmo, conđición que se acompaña normalmente de hiperventilación, la cual lleva a la pérdida de calor y deshidratación de las células de las vías respiratorias, aumentando así la osmolaridad intracelular y la liberación de mediadores inflamatorios, lo que finalmente lleva a una respuesta obstructiva exagerada (10-16).

Una de las primeras líneas terapéuticas que disminuyen el riesgo de exacerbación, es el uso de broncodilatadores de acción rápida o también llamados agonistas B adrenéroicos de acción corta, como el salbutamol. Tratamiento que tiene un nivel de evidencia tipo $C$ para el manejo del BIE, el que se recomienda sea aplicado de 2 a 4 inhalaciones de 15 a 20 minutos antes de empezar la actividad física, lo cual puede proporcionar una broncodilatación máxima en 15 a 60 minutos y una protección contra BIE durante al menos 3 horas en la mayoría de los pacientes (17-19). Todo lo anterior, sumado a un calentamiento previo de baja intensidad que produzca una leve sudoración, parece generar un período preventivo útil para disminuir el riesgo de BIE y le permite al sujeto asmático participar en igualdad de condiciones con aquellos que no padecen la enfermedad (20-22).

Otros autores como Tancredi et al. (23), evidenciaron una disminución deI VEF1 en sujetos asmáticos después de una prueba de ejercicio físico, realizada en una banda sin fin a una intensidad inferior del $80 \%$ de la frecuencia cardiaca máxima. Asimismo, Good et al. (24) muestran que en sujetos con BIE, una sesión de ejercicio físico de intensidad moderada $y$ de larga duración, reduce en igual proporción eI VEF1 al ser comparado con el ejercicio de alta intensidad y corta duración. O' neill et al. (25) evidenció que, en sujetos con hiperreactividad bronquial, el ejercicio interválico de alta intensidad genera una menor disminución deI VEF1 aI ser comparado con el continuo de larga duración.

Por lo anterior, no está completamente claro si los ejercicios prolongados de intensidad moderada pueden también ser considerados como desencadenantes del BIE. Siendo así, el presente estudio tiene como objetivo evaluar los cambios espirométricos producidos por el ejercicio físico continuo de intensidad moderada realizado en jóvenes no asmáticos y asmáticos broncodilatados.

\section{METODOLOGÍA}

Se realizó un estudio cuasi-experimental con una base poblacional de 10 estudiantes pertenecientes a la Facultad de Salud de la Universidad de Santander, los cuales fueron seleccionados por medio de un muestreo no probabilístico por conveniencia: cinco personas asmáticas y cinco personas no asmáticas. Como criterios de inclusión se estableció la participación voluntaria, ser mayor de 18 años, no presentar ninguna contraindicación relativa o absoluta para realizar EF y para el grupo de los casos, tener el diagnóstico médico de asma bronquial.

Los datos sociodemográficos y clínicos fueron registrados en un cuestionario autodiligenciado. Las principales variables dependientes fueron la relación CVF/VEF1 y el VEF1, su análisis se basó en los valores de referencia de Knudson. La variable independiente principal fue el EF moderado realizado y entre las variables antropométricas medidas, están la talla, la masa corporal total (MCT) y el índice de masa corporal (IMC).

El estudio se dividió en tres fases: 1) Socialización sobre los objetivos del estudio, firma del consentimiento informado. 2) Asiognación de los participantes a los grupos: asmáticos y no asmáticos. 3) Medición de la función pulmonar preejercicio. 4) Aplicación del protocolo de ejercicio. 5) Medición de la función pulmonar posejercicio (Figura 1).

La medición de la función pulmonar fue hecha mediante un espirómetro (WeIch Allyn Tipo ScHiller SP-1 RS-232), el protocolo usado se basó en las recomendaciones de las Asociación Americana de Tórax (12). Se registró una espirometría basal para ambos grupos y otra posbroncodilatador a los 15 minutos solo en el grupo de los casos (salbutamol 2 puffs del IDM con inhalocámara AeroChamber Plus). La técnica inhalatoria se realizó según los lineamientos del consenso Separ-Alat (26-27). Posteriormente, se dio inicio a la única sesión de ejercicio en ambos grupos, según el siguiente protocolo: 10 minutos de calentamiento que incluía ejercicios de las cuatro extremidades y deI tronco; 20 minutos de ejercicio en banda sin fin al $60 \%$ de la Frecuencia Cardiaca (FC) máxima según Tanáka (28), controlada por monitores de frecuencia cardíaca (Polar, Sounto, Omrom) y al finalizar, 5 minutos de 


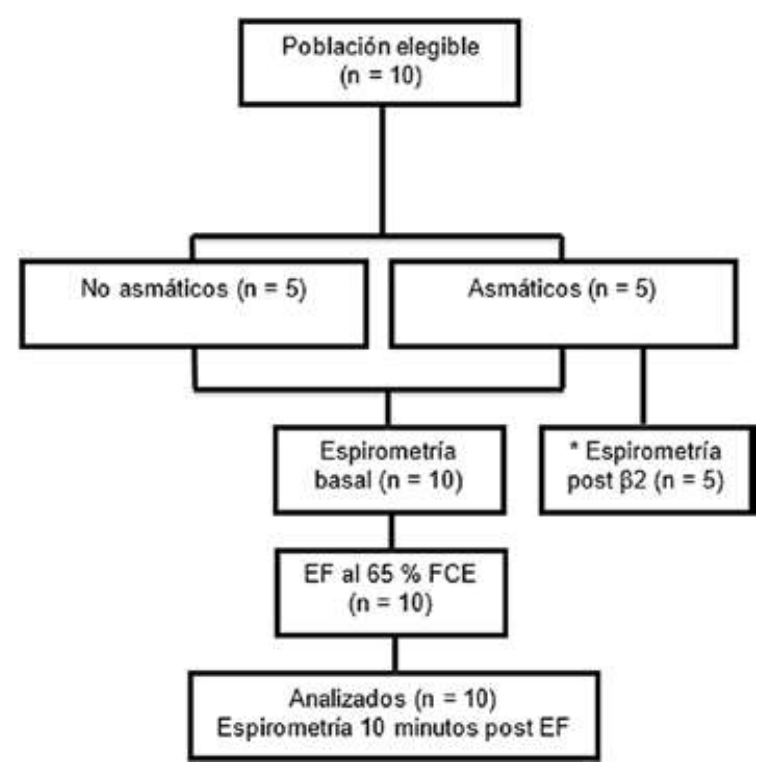

*Espirometría pos $\beta 2$ realizada únicamente a los pacientes asmáticos

Figura 1. Diagrama recolección de datos

recuperación activa. A los 10 minutos de finalizar el protocolo de EF, ambos recibieron una valoración espirométrica. El ejercicio se desarrolló en un solo día, a las diez de la mañana, en una temperatura ambiente 77 grados Fahrenheit y una humedad de 80 \%. El comité de investigaciones del programa de Fisioterapia de la Universidad de Santander aprobó la investigación para desarrollarse como proyecto de aula de la asiognatura Fisiología del ejercicio, con el acta n 23 del 2013.

\section{ANÁLISIS ESTADÍSTICO}

Los datos se analizaron en Stata 13,0. Para las medidas en escala numérica se calcularon mediana y rango intercuartílico y para las medidas en escala nominal, se calcularon frecuencias absolutas y relativas. La comparación por presencia o no de asma y la comparación de diferencias del pre y post ejercicio por la variable asma se realizó mediante la prueba rango, con sumas de Wilcoxon para muestras independientes. La distribución de sexo y uso de medicamentos según presencia o no de asma se realizó mediante el test exacto de Fisher. La comparación de diferencias dentro de cada grupo se realizó utilizando el test de Friedman para el grupo con asma y la prueba de rango con signos de Wilcoxon para el grupo sin asma. Se utilizaron modelos lineales generalizados usando una distribución gaussiana y la identidad como función de enlace para evaluar el efecto de la presencia de asma sobre los resultados espirométricos finales, ajustados por los resultados basales y peso corporal total.

\section{RESULTADOS}

La muestra estuvo conformada por 5 personas sin asma y 5 con asma con una mediana de 216 (RIC $=108-273$ ) meses de evolución. Estos últimos usaban como fármaco de rescate el salbutamol y solo el $60 \%$ de ellos la beclometasona como medicamento de control de la enfermedad. La talla y el IMC fue mayor en los sujetos asmáticos, la relación $\mathrm{VEF} 1 / \mathrm{CVF} 1$ (\%) fue superior en los sujetos no asmáticos (con asma: 89 (RIC $=87-97$ ) vs sin asma 109 (RIC $=97-109$ ); $\mathrm{p}=0,044$ ) y en general las variables clínicas basales no mostraron diferencias estadísticamente significativas (Tabla 1).

En la Tabla 2, se presentan los resultados espirométricos antes (evaluación basal) y después (evaluación final) del ejercicio e inmediatamente después del uso del broncodilatador para el grupo con asma. Al comparar los sujetos con y sin asma, no se encontraron diferencias significativas en la evaluación basal ni final, excepto para $\mathrm{VEF} 1 / \mathrm{CVF}$ (\%) en la evaluación basal. En las comparaciones dentro de cada grupo se encontró que en el grupo con asma no hubo diferencias estadísticamente siognificativas en las variables evaluadas en los tres momentos, y en el grupo sin asma no hubo diferencias relevantes al comparar la evaluación basal y final en ninguna de las variables de interés.

En la Tabla 3, se evidencian los cambios de la relación VEF1/CVF después del EF. Específicamente, Ios sujetos con asma presentaron un aumento de $6 \%$ y el grupo sin asma una disminución del $1 \%, p=0,036$.

Los resultados espirométricos post ejercicio según la presencia de asma, ajustados por evaluación basal y masa corporal total no fueron significativamente diferentes según la presencia o no de asma, excepto para CVF, en el cual se encontró una sionnificativa disminución en el grupo con asma (Tabla 4). 
Tabla 1. Características basales de la población de estudio, $\mathrm{n}=10$

\begin{tabular}{|c|c|c|c|}
\hline Variable & $\begin{array}{c}\text { Grupo con asma } n=5 \\
\text { Mediana (RIC)/n (\%) }\end{array}$ & $\begin{array}{l}\text { Grupo sin asma } n=5 \\
\text { Mediana }(\mathrm{RIC}) / \mathrm{n}(\%)\end{array}$ & Valor $p$ \\
\hline Edad (años) & $23(20,5-27,5)$ & $20(19,5-25,5)$ & $0,458^{*}$ \\
\hline Femenino & $4(57,1)$ & $3(42,9)$ & $1,000^{+}$ \\
\hline Masculino & $1(33,3)$ & $2(66,7)$ & \\
\hline Evolución (meses) & $216(108-273)$ & NA & NA \\
\hline Salbutamol & $5(100)$ & $0(0,0)$ & $0,008^{+}$ \\
\hline Beclometasona & $3(60)$ & $0(0,0)$ & $0,167^{+}$ \\
\hline Symbicort & $1(20)$ & $0(0,0)$ & $1,000^{+}$ \\
\hline Montelukast & $1(20)$ & $0(0,0)$ & $1,000^{+}$ \\
\hline PAS (mmHg) & $115(105-120)$ & $120(117,5-130)$ & $0,118^{*}$ \\
\hline $\mathrm{PAD}(\mathrm{mmHg})$ & $70(70-80)$ & $70(70-75)$ & $0,513^{*}$ \\
\hline FC (Ipm) & $88(70-98)$ & $82(68,5-88,5)$ & $0,251^{*}$ \\
\hline SatO2 (\%) & $98(97,5-98,5)$ & $98(97-98,5)$ & $0,650^{*}$ \\
\hline Talla (mts) & $1,70(1,65-1,75)$ & $1,65(1,62-1,70)$ & $0,203^{*}$ \\
\hline MCT (kg) & $70(60-75,1)$ & $56,3(52,5-57)$ & $0,047^{*}$ \\
\hline IMC (m/kg2) & $23,62(20,43-26,85)$ & $19,95(18,85-21,46)$ & $0,076^{*}$ \\
\hline VEF1/CVF & $0,76(0,62-0,84)$ & $0,95(0,82-1,00)$ & $0,076^{*}$ \\
\hline VEF1/CVF (\%) & $89(72,5-97)$ & $109(96-114)$ & $0,044^{*}$ \\
\hline VEF1(L/s) & $2,69(2,39-3,48)$ & $3,01(2,89-3,38)$ & $0,465^{*}$ \\
\hline VEF1 (\%) & $79(65,5-95,5)$ & $91(89-101,5)$ & $0,116^{*}$ \\
\hline CVF (L) & $3,84(3,17-4,90)$ & $3,21(3,00-3,95)$ & $0,347^{*}$ \\
\hline CVF \% & $96(80-98,5)$ & $87(83-91,5)$ & $0,465^{*}$ \\
\hline
\end{tabular}

*Prueba rango con sumas de Wilcoxon para muestras independentes. ${ }^{\dagger}$ Test exacto de Fisher. NA = No aplica. RIC: Rango Intercuartílico

Tabla 2. Variables clínicas durante el seguimiento, $\mathrm{n}=10$

\begin{tabular}{|c|c|c|c|c|c|c|c|c|c|c|}
\hline \multirow{3}{*}{ Variable } & \multirow{2}{*}{\multicolumn{2}{|c|}{ Evaluación basal }} & \multirow{3}{*}{ 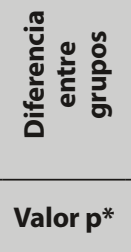 } & \multirow{2}{*}{\multicolumn{2}{|c|}{ Posbroncodilatador }} & \multirow{2}{*}{\multicolumn{2}{|c|}{ Evaluación final }} & \multirow{3}{*}{ 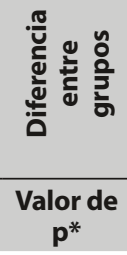 } & \multicolumn{2}{|c|}{$\begin{array}{l}\text { Diferencia en el } \\
\text { tiempo }\end{array}$} \\
\hline & & & & & & & & & $\begin{array}{c}\text { Con } \\
\text { asma }\end{array}$ & $\begin{array}{c}\text { Sin } \\
\text { asma }\end{array}$ \\
\hline & Con asma & Sin asma & & Con asma & $\begin{array}{c}\text { Sin } \\
\text { asma }\end{array}$ & Con asma & Sin asma & & $\begin{array}{l}\text { Valor } \\
\text { de } p^{+}\end{array}$ & $\begin{array}{l}\text { Valor } \\
\text { de } p^{\ddagger}\end{array}$ \\
\hline VEF1/CVF & $0,76(0,62-0,84)$ & $0,95(0,82-1,00)$ & 0,076 & $0,79(0,57-0,89)$ & NA & $0,80(0,65-0,88)$ & $0,95(0,83-0,96)$ & 0,117 & 0,449 & 0,500 \\
\hline $\begin{array}{c}\text { VEF1/CVF } \\
(\%)\end{array}$ & $89(72,5-97)$ & $109(96-114)$ & 0,044 & $92(67,5-102,5)$ & NA & $93(81-101)$ & $108(95,5-110)$ & 0,116 & 0,287 & 0,276 \\
\hline VEF1 (L/s) & $2,69(2,39-3,48)$ & $3,01(2,89-3,38)$ & 0,465 & $2,91(2,32-3,31)$ & NA & $2,82(2,45-3,55)$ & $2,98(2,91-3,33)$ & 0,465 & 0,549 & 0,500 \\
\hline VEF1 \% & $79(65,5-95,5)$ & $91(89-101,5)$ & 0,116 & $84(65-91)$ & NA & $83,4(67,6-97)$ & $91(87,5-101,5)$ & 0,251 & 0,549 & 0,494 \\
\hline CVF \% & $96(80-98,5)$ & $87(83-91,5)$ & 0,465 & $93(80-97,5)$ & NA & $92(77,8-98,5)$ & $90(82,5-93,5)$ & 0,754 & 0,493 & 0,586 \\
\hline
\end{tabular}

*Prueba rango con sumas de Wilcoxon para muestras independientes, comparación de sujetos con asma y sin asma

†Friedman test. „Wilcoxon signed-rank test. N.A= No aplica 
Tabla 3. Diferencias espirométricas del pre y post ejercicio según la presencia de asma, $\mathrm{n}=10$

\begin{tabular}{cccc}
\hline Variables & $\begin{array}{c}\text { Sin asma } \\
\text { Med (RIC) }\end{array}$ & $\begin{array}{c}\text { Con asma } \\
\text { Med (RIC) }\end{array}$ & $\begin{array}{c}\text { Valor } \\
\mathbf{p}^{*}\end{array}$ \\
\hline VEF1/CVF & $-0,01(-0,05 ; 0,01)$ & $0,03(0,03-0,05)$ & 0,117 \\
\hline $\begin{array}{c}\text { VEF1/CVF } \\
\text { (\%) }\end{array}$ & $-1,00(-6,00 ; 0,00)$ & $6,00(4,00-8,00)$ & 0,036 \\
\hline VEF1 (L/s) & $-0,03(-0,07 ;-0,01)$ & $0,08(0,04 ; 0,13)$ & 0,094 \\
\hline VEF1 \% & $-1,00(-2,00 ; 0,00)$ & $3,00(1,20 ; 4,00)$ & 0,074 \\
\hline CVF (L) & $0,02(-0,05 ; 0,16)$ & $-0,08(-0,11 ;-0,02)$ & 0,076 \\
\hline CVF \% & $0,00(-1,00 ; 3,00)$ & $-0,08(-0,11 ;-0,02)$ & 0,138 \\
\hline
\end{tabular}

Med: Mediana. RIC: Rango intercuartílico

*Luego intercuartílico. Two-sample Wilcoxon rank-sum test

\section{DISCUSIÓN}

Los resultados de esta investigación muestran que el ejercicio moderado de intensidad constante no modifica de forma aguda los parámetros espirométricos en sujetos sin asma o con asma broncodilatados. Lo anterior permite sugerir que realizar el EF con los parámetros aquí utilizados es seguro y no genera broncoespasmo, lo cual está en consonancia con lo encontrado en la revisión sistemática realizada por Carson et al. (29), quienes demostraron que el EF realizado a $<70 \%$ del $\nabla \mathrm{O}_{2}$ máx., es bien tolerado y debe ser hecho sin temor a las exacerbaciones.

Es importante resaltar que las posibilidades de presentar alteraciones en las variables espirométricas inducidas por el ejercicio en los asmáticos evaluados, pudieron ser disminuidas probablemente por la administración del broncodilatador antes de comenzar la sesión de $\operatorname{EF}(30,31)$. La anterior consideración se tuvo en cuenta a pesar de que los valores basales de la relación VEF1/CVF y el VEF1 indicaban que los sujetos asmáticos evaluados estaban controlados y sin limitación del flujo aéreo antes de que se les administrara el salbutamol a través de la inhalocámara.

Según la normativa de la Sociedad Española de Neumología y Cirugía Torácica (SEPAR), la mejoría deI VEF1 o en la CVF, son considerados sionnificativos,
Tabla 4. Resultados espirométricos post ejercicio según la presencia de asma

\begin{tabular}{|c|c|c|c|c|}
\hline Variable & Grupo & $\begin{array}{c}\text { *Beta } \\
\text { evaluación } \\
\text { final }\end{array}$ & IC95 \% & Valor $\mathbf{p}$ \\
\hline \multirow{2}{*}{ VEF1/CVF } & Sin asma & Ref & & \\
\hline & Con asma & 0,02 & $-0,09 ; 0,15$ & 0,651 \\
\hline \multirow{2}{*}{ VEF1 (L/s) } & Sin asma & Ref & & \\
\hline & Con asma & 0,04 & $-0,15 ; 0,24$ & 0,649 \\
\hline \multirow{2}{*}{ VEF1 \% } & Sin asma & Ref & & \\
\hline & Con asma & $-0,31$ & $-6,19 ; 5,56$ & 0,917 \\
\hline \multirow{2}{*}{ CVF (L) } & Sin asma & Ref & & \\
\hline & Con asma & $-0,25$ & $-0,45 ;-0,04$ & 0,017 \\
\hline
\end{tabular}

Ref: Grupo de referencia

*Beta ajustado por evaluación basal y masa corporal total. Modelo lineal generalizado

siempre y cuando, esta variable tenga un incremento $\geq 200 \mathrm{~mL}$ y del $12 \%, 15$ minutos posbroncodilatador (26). Teniendo en cuenta lo anterior, podemos deducir que no se observaron cambios significativos en los sujetos asmáticos después de aplicado el salbutamol. Asimismo, no existieron cambios significativos post-ejercicio en ninguno de los dos grupos, ya que para que esto suceda el VEF1 debe caer más del $10 \%$ con relación a la espirometría basal $(27,30)$. Otra posible razón por la cual no se observó una disminución clínicamente siognificativa deI VEF1 en el presente estudio fue la realización del protocolo de ejercicio en un ambiente controlado de temperatura $y$ humedad.

Por otra parte, se considera que la mayoría de BIE ocurren 10 minutos después de haber terminado el $\mathrm{EF}$, no obstante, existe una pequeña posibilidad de que este evento ocurra incluso 20 minutos después de terminar la sesión de entrenamiento, por lo cual se sugiere en futuras investigaciones proporcionar ambientes fríos y secos, así como un mayor seguimiento en el tiempo de las variables espirométricas (32).

Algunos autores como Tancredi et al. (23) y Good et aI. (24) han evidenciado la disminución del VEF1 en sujetos asmáticos o con antecedentes de BIE, después de realizar una sesión de entrenamiento continuo de 20 minutos de duración; sin embargo, es 
importante precisar que estos sujetos no recibieron broncodilatadores 8 horas antes de la sesión y que las intensidades utilizadas estuvieron cerca del $80 \%$ de la FC máxima.

O'Neill et al. (25) evidenciaron la respuesta aguda al ejercicio continuo e interválico de alta intensidad, concluyendo que este último generaba una menor disminución deI VEF1. Lo anterior puede llevar a pensar que los ejercicios prolongados de intensidad moderada también pueden ser considerados desencadenantes del BIE, a pesar de que en algunos estudios como el de França-Pinto et al. (33) argumentan que este tipo de entrenamiento realizado de forma regular, reduce la hiperreactividad bronquial, las citocinas proinflamatorias y las exacerbaciones en sujetos con asma moderada o grave.

Lo anterior hace pensar en la necesidad de generar futuras investigaciones que permitan comprender mejor el efecto de diferentes intensidades $y$ duraciones del ejercicio sobre la capacidad de respuesta de las vías respiratorias, teniendo en cuenta la gravedad del asma, el sexo y la condición física de los evaluados $(34,35)$, aspectos que no fueron tenidos en cuenta en este estudio, siendo considerados como parte de sus limitaciones, además del pequeño tamaño de muestra utilizado, lo que hace que los resultados no sean extrapolables a otras realidades.

Finalmente, se puede concluir que el EF, realizado bajo los parámetros sugeridos, es bien tolerado por los pacientes asmáticos evaluados, debido a que presenta bajo riesgo de broncoespasmo. Además su práctica regular puede mejorar de manera significativa la capacidad máxima de captación de oxígeno, incrementar el umbral anaeróbico y retardar la disnea durante el ejercicio.

\section{LIMITACIONES}

Dentro de las limitaciones del estudio están las producidas por el sesgo de selección, las cuales son propias del diseño utilizado. Por otra parte, la no inclusión de la severidad del asma, el sexo y la condición física en el análisis de los resultados impide detectar las diferentes respuestas que pueden ser condicionadas por estas variables. Todo lo anterior, sumado al tamaño de muestra utilizado, no permite que los resultados obtenidos sean generalizados. Finalmente, se sugiere para futuras investigaciones se incluya una muestra representativa y el control de variables antes registradas, con el objetivo de confirmar los beneficios o consecuencias sobre las variables espirométricas de este tipo de ejercicio físico.

\section{AGRADECIMIENTOS}

Los autores agradecen a la Facultad de Salud y al programa de Fisioterapia de la Universidad de Santander (UDES), por su apoyo y acompañamiento en la realización de la presente investigación.

\section{CONFLICTOS DE INTERESES}

Ninguno por declarar.

\section{REFERENCIAS BIBLIOGRÁFICAS}

1. Echegoyen A, Almudévar E, Urbiola E, et al. Eosinofilia en esputo versus óxido nítrico en aire exhalado: aplicación clínica en el asma. Anales Sis San Navarra. 2009;32(2):217-25.

2. Global Initiative for Asthma. Global Strategy for Asthma Management and Prevention [Internet]. Estados unidos: GINA; 2017 [cited Sep 23 de 2017]. Available from: file:///C:/Users/Informatica/Downloads/wms-GINA-2018-report- $71.3-002 \% 20(1)$.pdf

3. Pawankar R, Canonica GW, Holgate ST, Lockey RF. Allergic diseases and asthma: a major global health concern. Curr Opin Allergy Clin Immunol. 2012 Feb;12(1):39-41. DOI 10.1097/ACI.Ob013e32834ec13b.

4. National Heart, Lung and Blood Institute. Asthma [Internet]. Bethesda: NIH; 2017 [cited Sep 28 de 2017]. Available from: http://www nhlbi.nih.gov/health/dci/ Diseases/Asthma/Asthma 'WhatIs.htmI

5. Grupo Español para el Manejo del Asma. Guía española para el Manejo del Asma: para pacientes, padres y amigos [Internet]. Madrid: GEMA; 2005 [consultado Sep 28 de 2017]. Disponible en: https://issuu.com/separ/docs/gema

6. López Chicharro J, López Mojares LM. Fisiología clínica del ejercicio. Buenos Aires: Panamericana; 2008. 
7. Scott HA, Garg ML, Gibson PG, Wood LG. Asthma and inflammation. In: Manohar L Garg, Lisa G Wood, editors. Nutrition and Physical Activity in Inflammatory Diseases. Boston: CAB International; 2013. p. 416.

8. Aggarwal B, Mulgirigama A, Berend N. Exerciseinduced bronchoconstriction: prevalence, pathophysiology, patient impact, diagnosis and management. NPJ Prim Care Respir Med. 2018;28(1):31. DOI 10.1038/s41533-018-0098-2.

9. Drobnic F, Bordeiras L. Recomendaciones SEPAR sobre el cuidado del asma en condiciones extremas [Internet]. Barcelona: SEPAR; 2007 [consultado Sep 28 de 2017]. Disponible en: https://issuu.com/separ/ docs/normativa_separ_47

10. Escudero MP, Galindo M. Ejercicio físico y asma [Internet]. Madrid: YOU \& US; 2008 [consultado 23 Sept 2013]. Disponible en: http://pilarmartinescudero.es/ pdf/publicaciones/medicos/ejercicioyasma.pdf

11. Parsons JP, Hallstrand TS, Mastronarde JG, Kaminsky DA, Rundell KW, Hull JH, et al. An official American Thoracic Society clinical practice guideline: exercise-induced bronchoconstriction. Am J Respir Crit Care Med. 2013 May;187(9):1016-27. DOI 10.1164/ rccm.201303-0437ST.

12. Eichenberger PA, Diener SN, KofmehI R, Spengler CM. Effects of exercise training on airway hyperreactivity in asthma: a systematic review and metaanalysis. Sports Med. 2013 Nov;43(11):1157-70. DOI 10.1007/s40279-013-0077-2.

13. Mickleborough TD, Lindley MR, Ray S. Dietary salt, airway inflammation, and diffusion capacity in exercise-induced asthma. Med Sci Sports Exerc. 2005 Jun;37(6):904-14.

14. Scichilone N, Morici G, Zangla D, Arrigo R, Cardillo I, Bellia $\nabla$, et al. Effects of exercise training on airway closure in asthmatics. J Appl Physiol (1985). 2012 Sep;113(5):714-8. DOI 10.1152/japplphysiol.00529.2012.

15. Molis MA, Molis WE. Exercise-induced bronchospasm. Sports Health. 2010 Jul;2(4):311-7.

16. Avallone KM, McLeish AC. Asthma and aerobic exercise: a review of the empirical literature. J Asthma. 2013 Mar;50(2):109-16. DOI 10.3109/02770903.2012.759963.

17. De Zubiría Consuegra E, De Zubiría Salgado E, Alberto De Zubiría Salgado A. Asma bronquial, $2^{a}$ ed. Medellín: Panamericana; 2004.
18. Mendes FA, Almeida FM, Cukier A, Stelmach R, Jacob-Filho W, Martins MA, et al. Effects of aerobic training on airway inflammation in asthmatic patients. Med Sci Sports Exerc. 2011 Feb;43(2):197-203. DOI 10.1249/MSS.0bo13e3181edoea3.

19. Parsons JP. Exercise-induced bronchoconstriction. Otolaryngol Clin North Am. 2014 Feb;47(1):119-26. DOI 10.1016/j.otc.2013.09.003.

20. Severa, E. Sancho, J. Asma bronquial. Broncoespasmo inducido por el ejercicio. En: López C, López M. Fisiología Clínica del Ejercicio. Buenos aires:Editorial Médica Panamericana 2008. p. 139-147.

21. Morton AR, Fitch KD. Australian association for exercise and sports science position statement on exercise and asthma. J Sci Med Sport. 2011 Jul;14(4):312-6. DOI 10.1016/j.jsams.2011.02.009.

22. Riebe D, Ehrman J, Liguori G, Magal M; American College of Sports Medicine. ACSM's Guidelines for Exercise Testing and Prescription. 10 $0^{\text {th }}$ ed. Philadelphia: Wolters Kluwer.

23. Tancredi G, Quattrucci S, Scalercio F, De Castro G, Zicari AM, Bonci E, et al. 3-min step test and treadmill exercise for evaluating exercise-induced asthma. Eur Respir J. 2004 Apr;23(4):569-74.

24. Good J, Viana E, Burgomaster KA, Dogra S. Acute responses to sprint-interval and continuous exercise in adults with and without exercise-induced bronchoconstriction. J Sports Sci. 2018 Jun 20:1-9. DOI 10.1080/02640414.2018.1488520.

25. O’Neill C, Burgomaster K, Sanchez O, Dogra S. The acute response to interval and continuous exercise in adults with confirmed airway hyper-responsiveness. J Sci Med Sport. 2017 Nov;20(11):976-80. DOI 10.1016/j. jsams.2017.04.010.

26. García-Río F, Calle M, Burgos F, Casan P, Del Campo F, Galdiz JB, et al. Spirometry. Spanish Society of Pulmonology and Thoracic Surgery (SEPAR). Arch Bronconeumol. 2013 Sep;49(9):388-401. DOI 10.1016/j.arbres.2013.04.001.

27. Sociedad Española de Neumologia y Cirugia Toracica, Asociacion Latinoamericana de Torax. Consenso SEPAR-ALAT sobre terapia inhalada. Arch Bronconeumol. 2013;49(1):2-14.

28. Tanaka H, Monahan KD, Seals DR. Age-predicted maximal heart rate revisited. J Am Coll Cardiol. 2001 Jan;37(1):153-6. 
29. Carson KV, Chandratilleke MG, Picot J, Brinn MP, Esterman AJ, Smith BJ. Physical training for asthma. Cochrane Database Syst Rev. 2013 Sep;(9):CD001116. DOI 10.1002/14651858.CD001116.pub4.

30. Miller MR, Hankinson J, Brusasco V, Burgos F, Casaburi R, Coates A, et al. Standardisation of spirometry. Eur Respir J. 2005 Aug;26(2):319-38.

31. Rebelledo RC, Teixeira B, Correa C. Asma, estrés oxidativo y ejercicio físico: revisión de la literatura. Fisioterapia. 2014;36(1):40-8. DOI 10.1016/j. ft.2013.06.002.

32. Martin C, Moreno A. Prueba de broncoprovocación inducida por el ejercicio. An Pediatr Contin. 2009; 7(6):361-4.
33. França-Pinto A, Mendes FA, de Carvalho-Pinto RM, Agondi RC, Cukier A, Stelmach R, et al. Aerobic training decreases bronchial hyperresponsiveness and systemic inflammation in patients with moderate or severe asthma: a randomised controlled trial. Thorax. 2015 Aug;70(8):732-9. DOI 10.1136/thoraxjnI-2014-206070.

34. Hull JH, Ansley L, Price OJ, Dickinson JW, Bonini M. Eucapnic Voluntary Hyperpnea: Gold Standard for Diagnosing Exercise-Induced Bronchoconstriction in Athletes? Sports Med. 2016 Aug;46(8):1083-93. DOI 10.1007/s40279-016-0491-3.

35. Guenette JA, Witt JD, McKenzie DC, Road JD, Sheel AW. Respiratory mechanics during exercise in endurance-trained men and women. J Physiol. 2007 Jun;581 (Pt 3):1309-22. 